\title{
Langzeitstudie
}

\section{Das Diabetesrisiko mit "guten“ Pflanzen senken}

Eine pflanzliche Ernährung senkt das Risiko, einen Typ-2-Diabetes zu entwickeln, insbesondere dann, wenn sie reichlich hochwertige vegetarische Nahrungsmittel enthält. Darauf deutet eine Untersuchung mit über 200.000 Teilnehmern hin.

Wie Studien zeigen konnten, ist eine vegetarische Ernährung mit einem geringeren Risiko für Typ-2-Diabetes assoziiert. Bisher untersuchten Forscher aber vor allem das Diabetesrisiko reiner Vegetarier, das sie dann mit dem Risiko von Nicht-Vegetariern verglichen. In einer amerikanischen Studie haben Wissenschaftler um Ambika Satija der Harvard T.H. Chan School of Public Health (Boston) untersucht, ob es in Bezug auf das Diabetesrisiko auch eine Rolle spielt, welche vegetarischen Lebensmittel konsumiert werden.

\section{Ernährung anhand von Indizes bewertet}

Dazu analysierten die Forscher Daten aus drei unterschiedlichen Studien, in denen insgesamt über 200.000 US-amerikanische Männer und Frauen über mehr als 20 Jahre untersucht wurden. Die Teilnehmer füllten alle zwei bis vier Jahre Fragebögen zu ihren Ernährungsgewohnheiten, ihrem Lebensstil und ihren Erkrankungen aus.

Insgesamt wurden in den Fragebögen 130 Nahrungsmittel erfasst. Diese wurden dann den Kategorien „gesunde pflanzliche

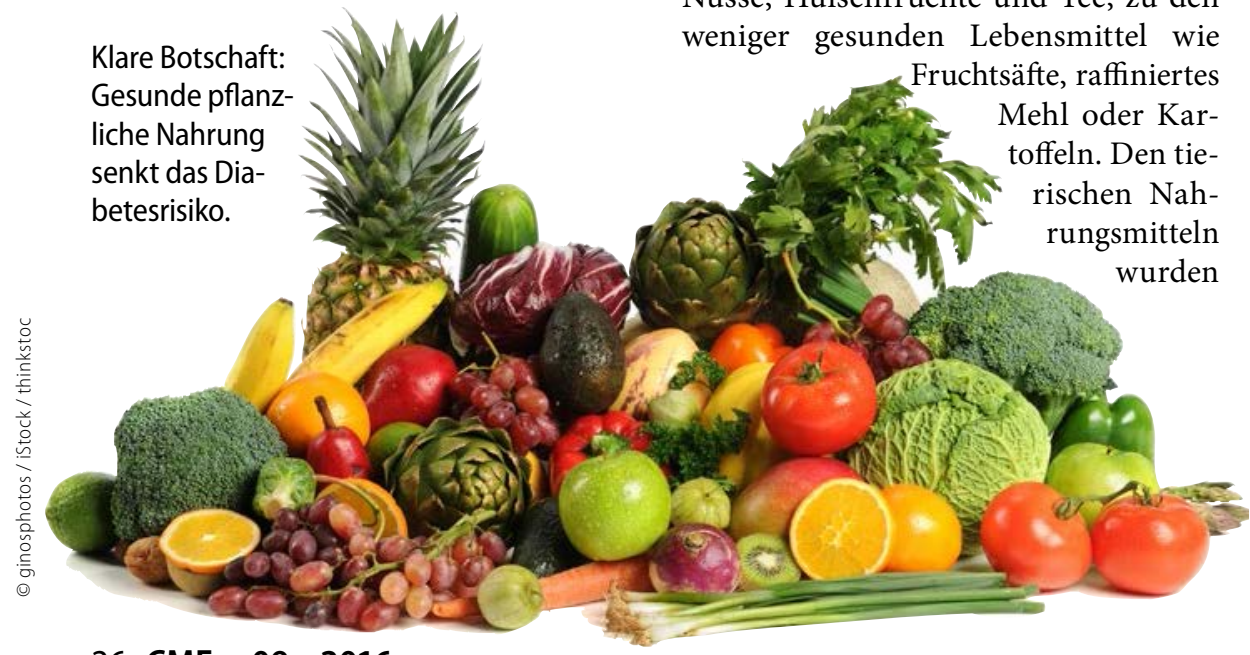

Nahrungsmittel“, „weniger gesunde pflanzliche Nahrungsmittel“ und „tierische Nahrungsmittel“ zugeordnet. Die Wissenschaftler bewerteten die Ernährung der Teilnehmer anhand von Indizes, bei denen Lebensmitteln aus der Kategorie "gesunde pflanzliche Nahrungsmittel“ höhere Punktzahlen, solchen aus der Kategorie „tierische Nahrungsmittel“ niedrigere Punktzahlen und schließlich solchen aus der Kategorie „weniger gesunde pflanzliche Nahrungsmittel“ je nach Fragestellung niedrige oder hohe Punktzahlen zugeteilt wurden. Die Punkte wurden für jeden Teilnehmer addiert, woraus sich dann sein Index ergab. Die Probanden wurden dann nach Indizes in Dezile eingeteilt.

Die zwei Gruppen „gesunde “ und „weniger gesunde pflanzliche Nahrungsmittel" bildeten die Forscher anhand von wissenschaftlichen Erkenntnissen zu dem Zusammenhang zwischen verschiedenen vegetarischen Lebensmitteln und Erkrankungen wie Typ-2-Diabetes, Krebs, Übergewicht, Bluthochdruck oder Dyslipidämie. Zu den gesunden pflanzlichen Nahrungsmitteln zählten sie unter anderem Vollkorngetreide, Obst, Gemüse, Nüsse, Hülsenfrüchte und Tee, zu den weniger gesunden Lebensmittel wie

Klare Botschaft: Gesunde pflanzliche Nahrung senkt das Diabetesrisiko.
Fleisch, Eier, Milchprodukte, Meeresfrüchte, Fisch und tierische Fette zugeordnet.

\section{Reduktion des Diabetesrisikos um 34\%}

Betrachteten die Wissenschaftler, wie häufig vegetarische Nahrungsmittel insgesamt konsumiert wurden, stellten sie fest, dass die Teilnehmer, die sich im höchsten Dezil befanden, ein 20\% geringeres Diabetesrisiko aufwiesen, als die, die sich im niedrigsten Dezil befanden (Hazard Ratio = 0,80, 95\%-Konfidenzintervall: 0,74-0,87). Wurde zusätzlich berücksichtigt, welche vegetarischen Nahrungsmittel konsumiert wurden - ob also häufig "gesunde pflanzliche Nahrungsmittel“ auf dem Speiseplan standen - hatten Teilnehmer, die sich im höchsten Dezil befanden, ein um 34\% reduziertes $\mathrm{Ri}$ siko für Diabetes mellitus Typ 2 im Vergleich $z u$ denen, die sich im niedrigsten Dezil befanden (Hazard Ratio $=0,66$, 95\%-Konfidenzintervall: 0,61-0,72). Die Teilnehmer aus dem höchsten Dezil hatten vier Portionen tierische Nahrungsmittel pro Tag zu sich genommen, während die Probanden des niedrigsten Dezils fünf bis sechs Portionen pro Tag konsumiert hatten. Das Diabetesrisiko unterschied sich also zwischen den beiden Gruppen um 34\%, obwohl der Konsum an tierischen Nahrungsmitteln in der Gruppe mit der gesündesten Ernährung nur geringfügig niedriger war.

Diese Ergebnisse unterstützen laut Studienautoren die bestehende Empfehlung, vermehrt „gesunde pflanzliche Nahrungsmittel“ zu konsumieren. Außerdem solle man seltener „weniger gesunde pflanzliche Nahrungsmittel " und tierische Nahrungsmittel zu sich nehmen, um das Diabetesrisiko zu reduzieren.

(Constance Jakob)

Satija A et al. Plant-Based Dietary Patterns and Incidence of Type 2 Diabetes in US Men and Women: Results from Three Prospective Cohort Studies. PLoS Med 2016; 13(6): e1002039; doi: 10.1371/journal.pmed.1002039 